\title{
Descripción de los pacientes con fibrilación auricular no valvular que ingresan al servicio de urgencias
}

\author{
Diego A. Pinto ${ }^{a}$, Carlos A. Sánchez-Vallejo ${ }^{b}$, Anderson López Pedraza ${ }^{c}$, \\ Erika P. Vergara ${ }^{d, *}$, Óscar A. Sáenz ${ }^{a}$, Francisco González ${ }^{d}$, \\ Juan C. Martínez-Acosta ${ }^{a}$, Paola Bonilla ${ }^{a}$ y Sergio Remolina ${ }^{e}$
}

\author{
a Hospital Santa Clara ESE, Bogotá, Colombia \\ b Universidad El Bosque, Bogotá, Colombia \\ ' Hospital Santa Clara ESE, Facultad de Medicina Universidad El Bosque, Bogotá, Colombia \\ d Epidemiología Clínica, Hospital Santa Clara ESE, Bogotá, Colombia \\ e Grupo de Investigación Servicio de Urgencias, Hospital Santa Clara ESE, Bogotá, Colombia
}

Recibido el 19 de junio de 2015; aceptado el 2 de septiembre de 2015

Disponible en Internet el 28 de octubre de 2015

\author{
PALABRAS CLAVE \\ Fibrilación auricular; \\ Accidente \\ cerebrovascular \\ agudo; \\ Agentes \\ anticoagulantes; \\ Warfarina
}

\begin{abstract}
Resumen
Introducción y objetivos: La fibrilación auricular es la arritmia cardiaca más frecuente y una de las complicaciones más temidas es la embolia sistémica y/o el accidente cerebrovascular (ACV). En la actualidad se cuenta con diversas terapias antitrombóticas para prevención del primero, sin embargo, hay temor por complicaciones relacionadas con la anticoagulación, y aunque existen recomendaciones de diferentes guías de prácticas clínicas, continúa el uso subóptimo de la misma. El objetivo del estudio es describir datos clínicos, diagnósticos y terapéuticos de pacientes con fibrilación auricular que ingresan al servicio de urgencias, además, se realizó una correlación entre el riesgo de embolia sistémica y de sangrado con el tipo de terapia recibida. Métodos: Estudio descriptivo retrospectivo tipo serie de casos de pacientes con diagnóstico de la fibrilación auricular no valvular durante un año. Se reportan las frecuencias de comorbilidades, diagnósticos de ingreso y tipo de terapia específica.

Resultados: Se incluyeron 98 pacientes, la media de edad fue 72 años, la falla cardiaca y la hipertensión arterial fueron las principales comorbilidades; el 92\% tenían indicación de
\end{abstract}

\footnotetext{
* Autor para correspondencia.

Correo electrónico: paoli1982@gmail.com (E.P. Vergara).
} 


\section{KEYWORDS}

Atrial fibrillation;

Acute

cerebrovascular

accident;

Anticoagulation

agents;

Warfarin anticoagulación por la escala $\mathrm{CHA}_{2} \mathrm{DS}_{2} \mathrm{VASc}, 54 \%$ de estos pacientes no la venían recibiendo a pesar de que se calculó en la mayoría bajo riesgo de sangrado. El 5\% de los pacientes ingresaron con diagnóstico de $\mathrm{ACV}$ isquémico y el $5 \%$ presentaban sobreanticogulación.

Conclusión: Las características de nuestra población con la fibrilación auricular es similar a la descrita en la literatura, pero existe baja adherencia a las recomendaciones terapéuticas en anticoagulación.

( 2015 Sociedad Colombiana de Cardiología y Cirugía Cardiovascular. Publicado por Elsevier España, S.L.U. Este es un artículo Open Access bajo la licencia CC BY-NC-ND (http:// creativecommons.org/s/by-nc-nd/4.0/).

\section{Description of patients with non-valvular atrial fibrillation admitted to the emergency room}

\begin{abstract}
Introduction and motivation: Atrial fibrillation is the most common cardiac arrhythmia. One of its most feared complications is the systemic embolism and/or the cerebrovascular accident (CVA) or stroke. Nowadays several antithrombotic therapies are available for the former; however, there are fears regarding complications related to anticoagulation, and even though recommendations of different clinical practice guidelines exist, blood-thinning drugs are still being underused. The goal of this study is to describe clinical, diagnostic and therapeutic data of patients admitted in the emergency room with atrial fibrillation; in addition, a correlation was made between the risk of systemic embolism and bleeding depending on the received treatment.

Methods: Retrospective, descriptive study of case series of patients diagnosed with non-valvular atrial fibrillation during a year. Comorbidity frequencies, admission diagnoses and specific therapy types are reported.

Results: 98 patients were included, average age was 72 years old, cardiac failure and high blood pressure were the main comorbidities; $92 \%$ showed an indication for anticoagulation therapy according to the $\mathrm{CHA}_{2} \mathrm{DS}_{2}$-VASc scale, $54 \%$ of those patients had not been receiving this therapy despite a low risk for bleeding was established for most of them. $5 \%$ of the patients received an admission diagnosis of ischemic stroke and $5 \%$ showed overanticoagulation.

Conclusion: The characteristics of our sample with atrial fibrillation are similar to those described in the literature, but there is a low adherence to the treatment recommendations for anticoagulation therapy.

(c) 2015 Sociedad Colombiana de Cardiología y Cirugía Cardiovascular. Published by Elsevier España, S.L.U. This is an open access article under the CC BY-NC-ND license (http:// creativecommons.org/licenses/by-nc-nd/4.0/).
\end{abstract}

\section{Introducción}

La fibrilación auricular es la arritmia cardiaca más frecuente, con una prevalencia de $1-2 \%$ de la población general. Esta cifra aumenta con la edad, con valores cercanos al $15 \%$ en pacientes mayores de 80 años ${ }^{1}$. Algunos estudios han demostrado que el riesgo de desarrollar la fibrilación auricular es alrededor de $25 \%$ a los 40 años de edad ${ }^{2}$. Lo anterior, sumado al envejecimiento de la población, conlleva a un aumento considerable en la cantidad de pacientes con esta patología ${ }^{3}$.

Los pacientes con la fibrilación auricular tienden a agrupar varias comorbilidades que afectan de manera significativa su pronóstico ${ }^{4}$. Entre las principales se encuentran: la hipertensión arterial, que constituye el principal factor de riesgo para fibrilación auricular y sus complicaciones; la falla cardiaca que está presente en $30 \%$ de los pacientes y puede ser una causa o consecuencia de la fibrilación auricular; la enfermedad valvular cardiaca presente en $30 \%$ de los casos y la enfermedad cardiaca isquémica, que se ha documentado en más del $20 \%$ de los pacientes ${ }^{5}$.

Una de las complicaciones más temidas de esta enfermedad es el aumento en el riesgo de la embolia sistémica y/o el accidente cerebrovascular (ACV). Estudios clásicos demostraron que cerca del $20 \%$ de todos los ACVs son secundarios a esta condición y que el riesgo también aumenta con la edad, alcanzando cifras mayores a $23 \%$ en pacientes mayores de 80 años de edad ${ }^{6}$. No obstante, estas cifras pueden estar subestimadas debido a un subdiagnóstico de esta condición en pacientes con la fibrilación auricular paroxística. Un estudio realizado por Gladstone y cols. demostró que en pacientes > 55 años de edad, con el ACV criptogénico, la tasa de detección de la fibrilación auricular puede aumentar cerca de un $13 \%$, si se utiliza una grabadora de eventos por 30 días posterior al evento ${ }^{7}$. Por tanto, aún desconocemos la verdadera magnitud del problema.

La gravedad del evento también es mayor en pacientes con el ACV de origen embólico secundario a la fibrilación 
auricular. Gatellari y cols. realizaron un estudio para evaluar los desenlaces de pacientes con el ACV y la fibrilación auricular en el cual encontraron que la mortalidad ajustada por la edad y las comorbilidades es mayor en pacientes con la fibrilación auricular que en pacientes con el ACV de cualquier otra etiología. Este efecto fue mayor a menor edad del paciente . $^{2}$.

La terapia antitrombótica con diversos agentes disponibles se ha convertido en una estrategia fundamental en el tratamiento de pacientes con la fibrilación auricular y el riesgo aumentado de los eventos cardioembólicos ${ }^{9,10}$. La warfarina se ha mantenido por muchos años como la primera opción de tratamiento para prevenir los eventos cardioembólicos en los pacientes con la fibrilación auricular, gracias a estudios que han demostrado $61-67 \%$ de reducción en el riesgo del $\mathrm{ACV}^{11}$. Otros estudios han demostrado que entre los pacientes tratados con antagonistas de la vitamina $\mathrm{K}$, lograr niveles de INR superiores a 2 no solo se asocia con reducción en la frecuencia del $\mathrm{ACV}$, sino que también está asociado con menor severidad y mortalidad relacionada con el $\mathrm{ACV}^{12}$.

Sin embargo, pese a la eficacia demostrada en los estudios clínicos, estos datos no son completamente reproducibles en la vida real. Uno de los aspectos más relevantes tiene que ver con el tiempo que pasan los pacientes en rango terapéutico de anticoagulación (INR 2-3). Un metaanálisis publicado en el año 2009, demostró que los pacientes que reciben la warfarina pasan sólo el 55\% del tiempo en rango terapéutico y que los pacientes de la comunidad pasan $11 \%$ menos tiempo en el rango terapéutico que los pacientes pertenecientes a las clínicas de anticoagulación ${ }^{13}$.

Este y otros factores, contribuyen a que exista una baja tasa de adherencia a las guías entre los médicos encargados del tratamiento de los pacientes con la fibrilación auricular. Un estudio realizado por Corin y cols. demostró que más del $30 \%$ de los pacientes con la fibrilación auricular y la indicación de terapia antitrombótica atendidos en un centro especializado permanecen subtratados o sin tratamiento, y que esta situación se asocia a mayor tasa del ACV y la mortalidad $^{14}$.

Con el fin de estandarizar la práctica médica y aumentar el uso de la terapia antitrombótica se han creado diversas escalas que sirven para predecir el riesgo de eventos cardioembólicos, especialmente el ACV en pacientes con la fibrilación auricular. El $\mathrm{CHA}_{2} \mathrm{DS}_{2} \mathrm{VASC}$ (falla cardiaca, hipertensión arterial, edad $>75$ años, diabetes, ACV, enfermedad vascular, edad entre 65-74 años, sexo femenino), es una escala que sirve para predecir el riesgo del ACV y definir la indicación de la anticoagulación. En la actualidad, se recomienda su utilización para estratificar el riesgo del $\mathrm{ACV}^{4}$.

Teniendo en cuenta lo anterior, se decidió llevar a cabo un estudio descriptivo para identificar las características clínicas de los pacientes que ingresan al servicio de urgencias de un centro de tercer nivel de atención de la ciudad de Bogotá con diagnóstico de la fibrilación auricular en un período de un año.

\section{Materiales y métodos}

Es un estudio descriptivo retrospectivo tipo serie de casos en el que se evaluó la población con un diagnóstico de fibrilación auricular no valvular que ingresó al servicio de urgencias de un centro de tercer nivel en la ciudad de Bogotá en el período comprendido entre noviembre de 2012 y noviembre de 2013. Se describió el riesgo tromboembólico a través de la escala $\mathrm{CHA}_{2} \mathrm{DS}_{2}$ VASc y el riesgo de sangrado con HASBLED. Se evaluó el estado de anticoagulación de los pacientes, la pertinencia de la terapia antitrombótica según las escalas y las complicaciones derivadas del tratamiento farmacológico.

\section{Participantes}

Todos los pacientes mayores de 18 años de edad, admitidos en el servicio de urgencias con diagnóstico de la fibrilación auricular no valvular, que ingresaron entre noviembre de 2012 y noviembre de 2013, fueron candidatos para ser incluidos en el estudio. Las características clínicas y demográficas de los pacientes, el estado de anticoagulación y los datos acerca de las escalas $\mathrm{CHA}_{2} \mathrm{DS}_{2}$ VASc y HASBLED se tomaron de las historias clínicas. Se excluyeron pacientes con la fibrilación auricular asociada a valvulopatia (insuficiencias o estenosis valvulares de origen reumático, válvulas protésicas o biológicas) y aquellos con antecedentes de ablación (pacientes que se espera están en seguimiento cardiológico estricto).

\section{Variables}

Las variables recopiladas de las historias clínicas fueron:

- Componentes individuales de la escala $\mathrm{CHA}_{2} \mathrm{DS}_{2}$ VASc: la falla cardiaca, la hipertensión arterial, la edad, la diabetes mellitus, el ACV isquémico previo, la enfermedad vascular, el sexo.

- Componentes individuales de la escala HASBLED: la hipertensión arterial no controlada, la alteración en la función renal o hepática, la edad, el ACV isquémico previo, el sangrado mayor previo o las condiciones que predispongan a sangrado, el INR lábil, los antecedentes farmacológicos que predispongan a sangrado y el consumo de alcohol.

- Consumo de anticoagulantes orales: INR al ingreso a urgencias en pacientes que tomaban antagonistas de la vitamina $\mathrm{K}$.

- Puntaje de $\mathrm{CHA}_{2} \mathrm{DS}_{2}$ VASc y HASBLED calculados.

- Complicaciones asociadas al tratamiento farmacológico o la ausencia de éste: los episodios de sangrado mayor y menor, el ACV isquémico.

\section{Análisis estadístico}

Se utilizaron los programas EXCEL y SPSS versión 21 para los análisis estadísticos.

Las variables cualitativas se agruparon en frecuencias y porcentajes, a las variables cuantitativas se les calculó las medias y las desviaciones estándar. A través del cálculo de las escalas, se identificaron pacientes con riesgo tromboembólico y riesgo de sangrado con anticoagulación. 


\section{Aspectos éticos}

De acuerdo a la Resolución No. 008430 de 1993 del Ministerio de Salud se considera una investigación sin riesgo ya que no se realizó intervención. El proyecto fue revisado y aprobado por el comité de investigación institucional.

\section{Resultados}

La tabla 1 muestra las características clínicas de los pacientes con fibrilación auricular que cumplían criterios de inclusión. En total se incluyeron 98 pacientes en el período de estudio. La distribución de género fue similar entre hombres y mujeres. Cerca del $84 \%$ de los pacientes era de raza mestiza y la media de edad fue de 72 años, aunque el intervalo fue bastante amplio. Las comorbilidades más frecuentes fueron: la hipertensión arterial $n=73(74,5 \%)$ y la falla cardiaca $n=59(60,2 \%) ; 12(12 \%)$ de los pacientes tenía antecedente del ACV de cualquier tipo. Cabe destacar que casi el 84\% de la población era mayor de 65 años de edad, lo que se relaciona con mayor riesgo del ACV y del sangrado.

Respecto al estado de anticoagulación, más de la mitad de los pacientes no estaba recibiendo la terapia antitrombótica (54\%). Entre los pacientes anticoagulados, 93\% recibían el antagonista de la vitamina $\mathrm{K}$ y $7 \%$ el dabigatran, tabla 1.

Tabla 1 Características clínicas y demográficas de los pacientes hospitalizados con diagnóstico de fibrilación auricular

\begin{tabular}{|c|c|c|}
\hline & n 98 & $\%$ \\
\hline \multicolumn{3}{|l|}{ CARACTERÍSTICAS DE LOS PACIENTES } \\
\hline Género & 98 & 100 \\
\hline Mujer & 50 & 51 \\
\hline Hombre & 48 & 49 \\
\hline \multicolumn{3}{|l|}{ Raza } \\
\hline Blanco & 15 & 15,3 \\
\hline Mestizo & 82 & 83,7 \\
\hline \multicolumn{3}{|l|}{ Edad PROMEDIO 72,7 años, rango (24 a 91) } \\
\hline$>75$ años & 48 & 49 \\
\hline 65-74 años & 34 & 34,7 \\
\hline$<65$ años & 16 & $16 \%$ \\
\hline Antecedente de falla cardiaca & 59 & 60,2 \\
\hline Hipertensión arterial no controlada & 64 & 65,3 \\
\hline Antecedente de diabetes mellitus & 14 & 14,3 \\
\hline Antecedente de ACV & 12 & 12,2 \\
\hline $\begin{array}{l}\text { Antecedente de enfermedad } \\
\text { vascular }\end{array}$ & 18 & 18,4 \\
\hline $\begin{array}{l}\text { Alteración en función renal } \\
\text { o hepática }\end{array}$ & 23 & 23,5 \\
\hline Antecedente de sangrado mayor & 6 & 6,1 \\
\hline INR lábil & 4 & 4,1 \\
\hline $\begin{array}{l}\text { Medicamentos que predisponen } \\
\text { a sangrado }\end{array}$ & 9 & 9,2 \\
\hline \multicolumn{3}{|l|}{ Anticoagulación } \\
\hline Ninguna & 53 & 54,1 \\
\hline Warfarina & 42 & 42,9 \\
\hline Dabigatran & 3 & 3,1 \\
\hline
\end{tabular}

Tabla 2 Diagnósticos de ingreso de los pacientes admitidos a urgencias con diagnóstico de fibrilación auricular

\begin{tabular}{lc}
\hline $\mathrm{n}$ & $(\%)$ \\
\hline Falla cardiaca & $27(27,6)$ \\
Fibrilación auricular & $25(25,5)$ \\
Evento coronario agudo & $9(9,2)$ \\
Sobreanticoagulación & $5(5,1)$ \\
ACV isquémico & $5(5,1)$ \\
Síncope & $5(5,1)$ \\
Dolor torácico & $3(3,1)$ \\
AlT & $3(3,1)$ \\
Shock cardiogénico & $1(1)$ \\
ACV hemorrágico & $1(1)$ \\
Otros* & $13(14,3)$ \\
Total & 98 \\
\hline
\end{tabular}

* Otros diagnósticos: neumonía, infección urinaria, EPOC entre otras.

Para el momento del estudio recién ingresaban al mercado otros medicamentos como el rivaroxaban y el apixaban.

\section{Diagnóstico y causa de ingreso a urgencias}

El principal motivo de hospitalización fue la falla cardiaca descompensada, que estaba presente en $27,6 \%$ de los pacientes. Síntomas y signos producto de la fibrilación auricular (las palpitaciones, la disnea y el dolor torácico), fueron motivo de la hospitalización en la cuarta parte de los pacientes. $5 \%$ de los pacientes ingresó con evidencia clínica o paraclínica de sobreanticoagulación y $5 \%$ de los pacientes ingresó por el ACV isquémico de cualquier severidad, tabla 2.

\section{Distribución de puntajes CHA2DS2VASc y HASBLED en la población}

Respecto a la distribución del $\mathrm{CHA}_{2} \mathrm{DS}_{2} \mathrm{VASc}$ en la población estudiada, encontramos que la mayoría de los pacientes (84\%), tenía un puntaje entre 2 y 5 puntos. El puntaje más frecuente fue $4(31,6 \%)$. Adicionalmente, $92 \%$ de los pacientes tenía puntaje de 2 o más, lo que implica que tenían indicación clara de anticoaguación, tabla 3.

Tabla 3 Distribución de puntaje CHA2DS2VASc en la población

\begin{tabular}{lcc}
\hline PUNTAJE CHA2DS2VASC & FRECUENCIA & PORCENTAJE \\
\hline 0 & 1 & 1 \\
1 & 6 & 6,1 \\
2 & 15 & 15,3 \\
3 & 17 & 17,3 \\
4 & 31 & 31,6 \\
5 & 19 & 19,4 \\
6 & 6 & 6,1 \\
7 & 3 & 3,1 \\
Total & 98 & 100 \\
\hline
\end{tabular}


Tabla 4 Distribución de puntaje HASBLED en la población

\begin{tabular}{lcc}
\hline HASBLED & FRECUENCIA & PORCENTAJE \\
\hline 0 & 5 & 5,1 \\
1 & 27 & 27,6 \\
2 & 39 & 39,8 \\
3 & 22 & 22,4 \\
4 & 4 & 4,1 \\
5 & 1 & 1 \\
Total & 98 & 100 \\
\hline
\end{tabular}

En relación a la escala HASBLED, encontramos que casi el $90 \%$ de los pacientes tiene puntajes entre 1 y 3 . El puntaje más frecuente fue 2 , que se encontró en el $40 \%$ de los pacientes. Respecto al riesgo de sangrado, encontramos que el $27 \%$ de los pacientes tenían un puntaje igual o mayor a 3 , que les confiere un alto riesgo de sangrado, tabla 4.

\section{Uso de anticoagulantes, indicación y rango de anticoagulación}

Para evaluar la adherencia a las recomendaciones internacionales, se midió el uso de anticoagulación, la calidad de acuerdo al INR y la relación entre anticoagulación y la indicación de estar anticoagulado.

Como se mencionó previamente, el $92 \%$ de los pacientes tenía indicación de anticoagulación según el puntaje de $\mathrm{CHA}_{2} \mathrm{DS}_{2}$ VASc. La tabla 5 relaciona el uso de anticoagulantes de acuerdo a la indicación de anticoagulación. Solo 5 pacientes $(5,1 \%)$ de la población en el estudio no tenían indicación de anticoagulación, de ellos 3 si recibían la warfarina. Sin embargo, el hallazgo más significativo consiste en que 51 pacientes $(54,8 \%)$ con indicación de anticoagulación por $\mathrm{CHA}_{2} \mathrm{DS}_{2} \mathrm{VASC}$ no estaba recibiendo ningún tratamiento anticoagulante. 42 pacientes (42\%) estaban en tratamiento con la warfarina y 3 (3\%) con el dabigatrán.

Entre los pacientes anticoagulados con la warfarina, 10 no tenían datos del INR días antes o al momento del ingreso a urgencias (pérdida de dato). El 17,3\% de los pacientes estaban subtratados, $10 \%$ tenían el INR supraterapéutico, y el $5 \%$ de los pacientes se encontraba en rango terapéutico de anticoagulación (tabla 6).

También se recolectaron datos acerca de los episodios de sangrados en pacientes con diagnóstico de fibrilación auricular, independiente del estado de anticoagulación y de acuerdo al riesgo de sangrado.

Ningún paciente clasificado como bajo riesgo presentó episodios de sangrado. Entre los pacientes que presentaban

Tabla 5 Relación entre indicación de anticoagulación y terapia antitrombótica

\begin{tabular}{lrclr}
\hline \multicolumn{5}{c}{ ANTICOAGULACIÓN } \\
\hline & NO & WARFARINA & NOAC & TOTAL \\
\hline INDICACION & & & & \\
NO & 2 & 3 & 0 & 5 \\
SI & 51 & 39 & 3 & 93 \\
TOTAL & 53 & 42 & 3 & 98 \\
\hline
\end{tabular}

Tabla 6 Rango de anticoagulación

\begin{tabular}{lcc}
\hline & Frecuencia & Porcentaje \\
\hline Sin anticoagulación & 53 & 54 \\
Warfarina & 42 & 42,8 \\
INR $<2$ & 17 & 17,3 \\
INR 2-3 & 5 & 5,1 \\
INR > 3 & 10 & 10,2 \\
Sin dato INR & 10 & 10,2 \\
Dabigatran & 3 & 3,1 \\
Total & 98 & 100 \\
\hline
\end{tabular}

* Se aclara que en 10 de los 42 pacientes anticoagulados con warfarina, no se tenía valor INR (pérdida de dato).

riesgo intermedio el 5\% tuvo al menos un episodio de sangrado; y entre los pacientes con riesgo alto, el $18,5 \%$ presentó algún episodio de sangrado. No se tienen datos del tipo de sangrado.

\section{Discusión}

Este estudio observacional descriptivo ofrece información sobre las características de los pacientes con la fibrilación auricular que ingresa al servicio de urgencias en un centro de tercer nivel en la ciudad de Bogotá. Los estudios epidemiológicos de la fibrilación incluyen principalmente, población caucásica ${ }^{4}$. Entre los 98 pacientes recolectados, la distribución del género fue simétrica y la edad promedio de los pacientes fue de 72 años, hallazgo que es de esperar teniendo en cuenta que la incidencia de la fibrilación auricular aumenta con la edad.

Uno de los estudios clásicos que describe las características de la población con fibrilación auricular en el Reino Unido encontró que la comorbilidad más frecuente era la hipertensión arterial, que estaba presente en el $37 \%$ de los pacientes, seguido de la cardiopatía isquémica y la valvular ${ }^{15}$. Nuestros datos son similares, pues encontramos que las dos comorbilidades más frecuentes en la población eran: la hipertensión arterial y la falla cardiaca. No obstante, la prevalencia de la hipertensión arterial entre nuestros pacientes fue casi el doble a lo documentado en la literatura y encontramos una elevada tasa de la hipertensión arterial no controlada (sistólica $>160 \mathrm{mmHg}$ ). En nuestra serie de casos la falla cardiaca fue la principal causa de ingreso a urgencias en los pacientes con la fibrilación auricular.

Más de la mitad de los pacientes (54\%) no estaba recibiendo anticoagulación, situación que ha sido demostrada en varios estudios alrededor del mundo. Lip y cols., en el año 1997 mostraron que sólo el 36\% de los pacientes recibía la warfarina, mientras que un $18 \%$ recibía el ácido acetil salicílico, a pesar que sólo el $12 \%$ de los pacientes tenía alguna contraindicación para ser anticoagulados ${ }^{15}$.

Esta baja tasa de uso de la terapia antitrombótica puede ser explicada por la antigüedad del estudio, dado que en la época la recomendación de anticoagular no era tan fuerte. Sin embargo, registros recientes muestran también que hay una preocupante subutilización de la warfarina entre los pacientes con la fibrilación auricular. Un gran registro nacional en Estados Unidos publicado por Waldo y cols. mostró que sólo el $55 \%$ de los pacientes con indicación de 
anticoagulación la estaban recibiendo ${ }^{16}$. Otros estudios, como el Euro Heart Survey on atrial fibrillation, han reportado el uso de anticoagulación en solo el $67 \%$ de la población ${ }^{17}$.

En nuestra serie de casos encontramos que el $54 \%$ de los pacientes con indicación de anticoagulación no la estaban recibiendo, esto indica que existe una baja adherencia a las recomendaciones de las guías sobre el uso de anticoagulantes en pacientes con fibrilación auricular y que estas cifras son comparables a la población mundial. Es importante mencionar el tipo de la población que se maneja en nuestra institución, en su mayoría ancianos, con pobre soporte social, algunos institucionalizados y con dificultades para acceder a los servicios de salud lo que genera temor al médico tratante para el inicio de la anticoagulación.

Un estudio publicado en el año 2008 por Gladstone y cols., en el que se evaluaron las características clínicas de los pacientes con el ACV secundario a la fibrilación auricular se encontró que sólo el $40 \%$ recibía la warfarina; y entre estos, el $74 \%$ de los pacientes se encontraba en rango subterapéutico de anticoagulación $(\text { INR }<2)^{18}$. En nuestro estudio logramos documentar que el $17 \%$ de los pacientes tenía un INR $<2$. No obstante, es más preocupante encontrar que no había registros de INR en el 63\% de los pacientes, lo que indica que estos pacientes no recibían anticoagulación, o en caso de recibirla, no estaban siendo monitorizados adecuadamente. Estos resultados guardan relación con muchos estudios que demuestran tasas subóptimas de uso adecuado de la terapia antitrombótica entre los pacientes ${ }^{19,20}$.

Estos datos resultan preocupantes, pues los estudios han demostrado que hasta el $64 \%$ de los ACVs en pacientes con fibrilación auricular ocurren con el INR $<2^{21}$. Por tanto, los pacientes incluidos en nuestro estudio tenían un riesgo especialmente alto de desarrollar el ACV. En este estudio encontramos que el $5 \%$ de los pacientes con fibrilación auricular ingresaron a urgencias por el ACV isquémico, dato que es ligeramente superior al $3 \%$ reportado en la literatura ${ }^{21}$. Un metaanálisis reciente evaluando la calidad de la anticoagulación documentó que los pacientes que toman la warfarina pasan solo la mitad del tiempo en rango terapéutico; así mismo, lograron determinar que medidas simples como ser tratado en una clínica de anticoagulación aumenta el porcentaje de tiempo en rango terapéutico del $51 \%$ a $63 \% 22$. Adoptar este tipo de medidas no solo reduce la tasa del ACV isquémico, también, se asocia a una disminución en la mortalidad por todas las causas cercana al $31 \%{ }^{21}$.

La mayoría de nuestros pacientes tenía un puntaje de $\mathrm{CHA}_{2} \mathrm{DS}_{2}$ VASc entre 2 y 5 , con más del $90 \%$ de los pacientes con puntaje mayor de 2 . Estos valores, son más altos que aquellos documentados en otros estudios. En el estudio original publicado por Gage y cols., donde se validó el $\mathrm{CHADS}_{2}$, solo el $66 \%$ de los pacientes tenía un puntaje mayor a $2^{23}$. De manera similar, el estudio original de $\mathrm{CHA}_{2} \mathrm{DS}_{2}$ VASC publicado en el año 2010 por Lip y cols., documentó que la mayoría de los pacientes tenía un puntaje entre 1 y 4 , y que el $74 \%$ de los pacientes tenía un puntaje mayor a $2^{24}$.

No es claro por qué razón nuestra población tiene puntajes mayores en las escalas de riesgo trombótico que otras poblaciones. Sin embargo, este hallazgo es muy relevante si se tiene en cuenta que a pesar de tener un mayor riesgo del ACV, la tasa de anticoagulación en nuestros pacientes fue igual de baja que la documentada en la literatura. Nuestros resultados son comparables con aquellos documentados en un registro italiano en el que se evaluaron pacientes con el ACV isquémico o hemorrágico asociados a la fibrilación auricular, en el que se documentó que en los pacientes que recibían cualquier tipo de terapia antitrombótica, el $\mathrm{CHA}_{2} \mathrm{DS}_{2}$ VASc promedio era de 5 puntos ${ }^{25}$.

En cuanto al riesgo de sangrado, se evaluó el puntaje en la escala del HASBLED al ingreso a urgencias. Encontramos que casi el $90 \%$ de los pacientes tiene puntajes entre 1 y 3 . Sólo el $27 \%$ cumplían la definición de riesgo alto de sangrado (puntaje igual o mayor a 3). Estos resultados son comparables con aquellos descritos en el estudio original publicado por Pisters y cols., del cual se derivó el HASBLED ${ }^{26}$. En su estudio encontraron que el $98 \%$ de los pacientes tiene puntajes entre 0 y 3 puntos. No obstante, en ese estudio se encontró que menos del $2 \%$ de los pacientes cumplían la definición del alto riesgo de sangrado, datos que son muy inferiores a los documentados en nuestra población. Es posible que esta percepción aumentada del riesgo de sangrado en nuestros pacientes haya contribuido en alguna manera a la baja tasa de anticoagulación, no obstante, no podemos demostrarlo a partir de este estudio. Finalmente, encontramos una buena correlación entre el riesgo predicho de sangrado por el HASBLED y la tasa de sangrados en nuestra población, con tasas cercanas al $20 \%$ en pacientes clasificados como riesgo alto. Sin embargo, nuestros datos no especifican el tipo de sangrado y no podemos determinar si se trataban de sangrados mayores.

\section{Conclusiones}

Este estudio descriptivo evaluó las características clínicas de los pacientes con la fibrilación auricular admitidos en el servicio de urgencias de un centro de tercer nivel en la ciudad de Bogotá. Las principales comorbilidades de los pacientes con la fibrilación auricular son: la hipertensión arterial y la falla cardiaca. Aún existe una baja adherencia a las guías de la fibrilación auricular, y la mayoría de los pacientes no reciben la anticoagulación, sin que exista contraindicación en la mayoría de los casos. Esta baja tasa de la anticoagulación permanece a pesar de que la mayoría de los pacientes tienen riesgo bajo del sangrado y riesgo alto del tromboembolismo de acuerdo a las escalas validadas para tal fin. Incluso en los pacientes anticoagulados, el seguimiento y el grado de control de la anticoagulación permanece subóptimo. Este estudio tiene limitaciones importantes. Primero, al ser un estudio descriptivo hecho a partir de pacientes que ingresaron a urgencias; puede haber un sesgo de selección al no contemplar la situación de los pacientes que se encuentran en manejo por consulta externa con diagnóstico de la fibrilación auricular, en quienes las tasas de la anticoagulación pueden ser mayores. Segundo, no se discriminaron los tipos del sangrado al evaluar la relación con el puntaje del HASBLED. Sin embargo, consideramos que la información recopilada es útil para hacerse una idea de la situación actual de las comorbilidades y la anticoagulación de los pacientes que tienen la fibrilación auricular y consultan a urgencias en nuestro medio. 


\section{Reconocimientos y agradecimientos}

Servicio de Urgencias Adultos Hospital Santa Clara ESE, Bogotá, Colombia.

\section{Responsabilidades éticas}

Protección de personas y animales. Los autores declaran que para esta investigación no se han realizado experimentos en seres humanos ni en animales.

Confidencialidad de los datos. Los autores declaran que en este artículo no aparecen datos de pacientes.

Derecho a la privacidad y consentimiento informado. LoS autores declaran que en este artículo no aparecen datos de pacientes.

\section{Conflictos de intereses}

El grupo declara no tener ningún conflicto de intereses.

\section{Bibliografía}

1. Stewart S, Hart CL, Hole DJ, MCMurray JJ. Population prevalence, incidence, and predictors of atrial fibrillation in the Renfrew/Paisley study. Heart. 2001;86:516-21.

2. Lloyd-Jones DM, Wang TJ, Leip EP, Larson MG, Levy D, Vasan RS, et al. Lifetime risk for development of atrial fibrillation: the Framingham Heart Study. Circulation. 2004;110:1042-6.

3. Alberts Eikelboom, Hankey. Antithrombotic therapy for stroke prevention in non-valvular atrial fibrillation. The Lancet Neurology. 2012;11(12):1066-81.

4. Camm AJ, Lip GY, De Caterina R, Savelieva I, Atar D, Hohnloser S, et al., ESC Committee for Practice Guidelines (CPG). 2012 focused update of the ESC guidelines for the management of atrial fibrillation: an update of the 2010 ESC guidelines for the management of atrial fibrillation. Developed with the special contribution of the European Heart Rhythm Association. Eur Heart J. 2012;33(21):2719-47.

5. Nabauer M, Gerth A, Limbourg T, Schneider S, Oeff M, Kirchhof P, Goette A, Lewalter T, et al. The Registry of the German Competence NETwork on Atrial Fibrillation: patient characteristics and initial management. Europace. 2009;11:423-34.

6. Wolf PA, Abbott RD, Kannel WB. Atrial fibrillation as an independent risk factor for stroke: the Framingham Study. Stroke. 1991;22(8):983-8.

7. Gladstone D, Spring M, Dorian P, Panzov V, Thorpe E, Judith M, et al. Atrial fibrillation in patients with cryptogenic stroke. N Engl J Med. 2014;370(26):2467-77.

8. Gattellari M, Goumas C, Aitken R, Worthington JM. Outcomes for patients with ischaemic stroke and atrial fibrillation: the PRISM study (A Program of Research Informing Stroke Management). Cerebrovasc Dis. 2011;32(4):370-82.

9. Stroke prevention in atrial fibrillation study. final results. Circulation. 1991;84(2):527-39.

10. Hart RG, Benavente O, McBride R, Pearce LA. Antithrombotic therapy to prevent stroke in patients with atrial fibrillation: A meta-analysis. Ann Intern Med. 1999;131:492-501.
11. Owen. Antithrombotic treatment for the primary prevention of stroke in patients with non valvular atrial fibrillation: A reappraisal of the evidence and network meta analysis. Inter J Card. 2010;142(3):218-23.

12. Hylek EM, Go AS, Chang Y, Jensvold NG, Henault LE, Selby JV, Singer DE. Effect of intensity of oral anticoagulation on stroke severity and mortality in atrial fibrillation. N Engl J Med. 2003;349:1019-26.

13. Bloomfield HE, Krause A, Greer N, Taylor B, MacDonald R, Rutks I, et al. Meta-analysis: Effect of patient self-testing and selfmanagement of long-term anticoagulation on major clinical outcomes. Ann Intern Med. 2011;154(7):472-82.

14. Gorin. Prognosis and guideline-adherent antithrombotic treatment in patients with atrial fibrillation and atrial flutter. CHEST Journal. 2011;140(4):911.

15. Lip GY, Golding DJ, Nazir M, Beevers DG, Child DL, Fletcher RI. A survey of atrial fibrillation in general practice: The west birmingham atrial fibrillation project. $\mathrm{Br} \mathrm{J}$ gen Pract. 1997;47(418).

16. Waldo AL, Becker RC, Tapson VF, Colgan KJ. Hospitalized patients with atrial fibrillation and a high risk of stroke are not being provided with adequate anticoagulation. J Am Coll Cardiol. 2005;46:1729-36.

17. Kirchhof P, Ammentorp B, Darius H, De Caterina R, Le Heuzey J, Schilling R, et al. Atrial fibrillation management: a prospective survey in ESC member countries: the Euro Heart Survey on Atrial Fibrillation. Eur Heart J. 2005;26:2422-34.

18. Gladstone DJ, Bui E, Fang J, Laupacis A, Lindsay MP, Tu JV, et al. Potentially preventable strokes in high-risk patients with atrial fibrillation who are not adequately anticoagulated. Stroke. 2008;40(1):235-40.

19. Albers GW, Yim JM, Belew KM, Bittar N, Hattemer CR, Phillips BG, Kemp S, Hall EA, Morton DJ, Vlasses PH. Status of antithrombotictherapy for patients with atrial fibrillation in university hospitals. Arch Intern Med. 1996;156:2311-6.

20. Paciaroni M, Agnelli G, Caso V, Venti M, Milia P, Silvestrelli G, Parnetti L, Biagini S. Atrial fibrillation in patients with first-ever stroke: frequency, antithrombotic treatment before the event and effect on clinical outcome. J thromb Haemost. 2005;3(6):1218-23.

21. Go AS, Hylek EM, Chang Y, Phillips KA, Henault LE, Capra AM, et al. Anticoagulation therapy for stroke prevention in atrial fibrillation: How well do randomized trials translate into clinical practice? Jama. 2003;290(20):2685-92.

22. William L. Baker, Deborah A. Cios, Stephen D. Sander, Craig I. Coleman. Meta-analysis to assess the quality of warfarin control in atrial fibrillation patients in the United States. J Manag Care Pharm. 2009;15(3)

23. Gage BF, Waterman AD, Shannon W, Boechler M, Rich MW, Radford MJ. Validation of clinical classification schemes for predicting stroke: results from the National Registry of Atrial Fibrillation. JAMA. 2001;285:2864-70.

24. Lip GY, Nieuwlaat R, Pisters R, Lane DA, Crijns HJ. Refining clinical risk stratification for predicting stroke and thromboembolism in atrial fibrillation using a novel risk factor-based approach: the Euro Heart Survey on atrial fibrillation. Chest. 2010;137:263-72.

25. Palareti Salomone Cavazza. Stroke/thromboembolism and intracranial hemorrhage in a real-world atrial fibrillation population. CHEST Journal. 2014;146(4):1073.

26. Pisters. A novel user-friendly score (HAS-BLED) to assess 1-year risk of major bleeding in patients with atrial fibrillation. CHEST Journal. 2010;138(5):1093. 